archives-ouvertes

\title{
Micrometer scale resolution of materials by stray-field Magnetic Resonance Imaging
}

Maxime Van Landeghem, Bruno Bresson, Bernhard Blümich, Jean-Baptiste d'Espinose

\section{To cite this version:}

Maxime Van Landeghem, Bruno Bresson, Bernhard Blümich, Jean-Baptiste d'Espinose. Micrometer scale resolution of materials by stray-field Magnetic Resonance Imaging. Journal of Magnetic Resonance, Elsevier, 2011, 211 (1), pp.60 - 66. 10.1016/j.jmr.2011.04.002 hal-03168647

\section{HAL Id: hal-03168647 https://hal.archives-ouvertes.fr/hal-03168647}

Submitted on 13 Mar 2021

HAL is a multi-disciplinary open access archive for the deposit and dissemination of scientific research documents, whether they are published or not. The documents may come from teaching and research institutions in France or abroad, or from public or private research centers.
L'archive ouverte pluridisciplinaire HAL, est destinée au dépôt et à la diffusion de documents scientifiques de niveau recherche, publiés ou non, émanant des établissements d'enseignement et de recherche français ou étrangers, des laboratoires publics ou privés. 


\title{
Micrometer scale resolution of materials by stray-field Magnetic Resonance Imaging
}

\author{
Maxime Van Landeghem ${ }^{\mathrm{a}, \mathrm{b}, \mathrm{c}}$, Bruno Bresson ${ }^{\mathrm{a}}$, Bernhard Blümich ${ }^{\mathrm{b}}$, Jean-Baptiste d'Espinose de Lacaillerie ${ }^{\mathrm{a}, *}$ \\ ${ }^{a}$ Soft Matter Sciences and Engineering Laboratory, UMR 7615 CNRS UPMC, ESPCI ParisTech, 10 rue Vauquelin, 75005 Paris, France \\ ${ }^{\mathrm{b}}$ Institute of Technical and Macromolecular Chemistry, RWTH Aachen University, D-52056 Aachen, Germany \\ ${ }^{\mathrm{c}}$ Saint-Gobain Recherche, 39 quai Lucien Lefranc, BP 135, 93303 Aubervilliers, France
}

\section{A R T I C L E I N F O}

\section{Article history:}

Received 27 January 2011

Revised 30 March 2011

Available online 12 April 2011

\section{Keywords}

STRAFI

Inhomogeneous field

Fringe field

Static gradients

High resolution

MRI

\begin{abstract}
A B S T R A C T
As Magnetic Resonance Imaging devices are becoming more and more powerful, resolutions as small as $10 \mu \mathrm{m}$ can now be obtained. But, this is only possible when systems with slow transverse relaxation rates, like living tissues, are investigated. In this case, the time available for gradients space-encoding is long, and high $k$ values can be reached in the Fourier domain. However, numerous materials have fast relaxation rates, thus limiting the spatial resolution to a few hundreds of microns. The Stray Field Imaging technique has solved this problem by using a very high (typically $5000 \mathrm{G} / \mathrm{cm}$ ) static gradient. Consequently, the trajectory in $k$ space is accelerated and it is possible, in principle, to reach a micrometer resolution in a few hundreds of microseconds. Most of the time, however, only resolutions in the millimeter range can be achieved due to mispositioning of the sample within the static magnetic field. Here, we show that by finely mapping the magnetic field and precisely positioning the sample, it is possible with a standard spectrometer to reach a micrometer resolution even on very fast relaxing materials.
\end{abstract}

\section{Introduction}

Magnetic Resonance Imaging (MRI) [1,2] is now used worldwide as a routine medical diagnostics tool. The attractiveness of this method is the use of three dimensional pulsed field gradients in order to scan k-space. After multi-dimensional Fourier transformation, three dimensional images are obtained with a typical resolution of $100 \mu \mathrm{m}$, and even in the range of $10 \mu \mathrm{m}$ in the most favorable cases [3]. Although this technique is very efficient in providing images of soft materials like living tissues [4], the use of pulse field gradients is less suitable in materials science. Indeed, solid materials are often characterized by short $T_{2}$ 's and can have strong internal field gradients. This makes the imaging process far more difficult.

The Stray Field Imaging (STRAFI) method originally proposed by Samoilenko [5] circumvents some of the limitations of conventional MRI by using the high static magnetic field gradients surrounding conventional super-conducting NMR or MRI magnets. These stable stray field gradients are sufficient to overcome the broad linewidths of solid materials and reliable 1D images of hard matter can be obtained by profiling the sample. The static gradients are much higher (typically $50 \mathrm{~T} / \mathrm{m}=5000 \mathrm{G} / \mathrm{cm}$ ) than the ones which can be commonly reached in a pulsed mode. STRAFI has typically allowed planar samples to be profiled with a resolu- tion from a millimeter [6] to a few hundreds micrometers [7], even in materials with very short $T_{2}$ 's. In particular, this method has been used to study protons in solid matter such as ice [8], resins [6] and polyacrylates [9] as well as in the interstitial phase of porous materials like cements [10,11] or ceramics [12]. Moreover, as in MRI, the gradient can be also used to encode the displacement of spins and measure diffusion coefficients [13,14].

The resolution of STRAFI profiling is limited by the fact that the stray field gradient is not constant over the volume of interest and, obviously, neither its direction nor its intensity can be corrected since the field is static. Therefore, the excited volume is often curved, and, in practice, the resolution is only in the millimeter range despite the nominal strength of the gradient. Previous studies have relied on the rough field plots provided by manufacturers of commercial magnets to optimize the resolution [5,15-17] but still, high resolution remains difficult to achieve in this manner. Here, we show that by mapping finely the magnetic field, it is possible to find a region where the curvature of the field is low enough to reach micron resolution on samples as wide as a few centimeters. Furthermore, in this region the gradient strength remains high enough for STRAFI studies of materials with short relaxation times.

\section{Theory}

In this section, the principle of the STRAFI experiment is presented. The equations which allow to evidence and to precisely locate the STRAFI plane are numerically solved. 


\subsection{Principle of the STRAFI experiment}

The STRAFI principle is fully described by McDonald [18-20], so only a brief outlines is needed here. In a STRAFI experiment, the sample is placed in the fringe or stray field $\vec{H}_{G}$ of gradient $\vec{G}$ surrounding the central homogeneous region of a large superconducting magnet, spreading the Larmor frequencies $\omega$ of the sample's protons according to their positions $\vec{r}$ following the well-known equation of MRI

$\omega \vec{r}=\gamma\left|\vec{H}_{G}(\vec{r})\right|=\gamma \vec{G} \cdot \vec{r}+\omega_{0}$

Then, a selective radio-frequency pulse of frequency $\omega_{0} / 2 \pi$ and finite length $t_{\mathrm{p}}$ is applied. It excites only the nuclear spins within the isofield slice defined by the above equation, with a thickness $\Delta s$ determined by the bandwidth according to (for a square pulse)

$\Delta s=\frac{\Delta \omega}{\gamma G}=\frac{2 \cdot \pi t_{\mathrm{p}}}{\gamma G}$

The recorded signal is directly proportional to the number of spins inside the selected slice. Afterwards, the sample is mechanically moved perpendicularly to the slice by a stepper motor, and the process is repeated to complete a one-dimensional profile. Actually, because the profile is performed along a single axis, the resolution is not limited by the bandwidth of the pulse but mainly by the curvature of the selected slice. As a consequence, the ideal slice must be planar. In what follows, this planar slice is called the STRAFI plane.

\subsection{Existence of a STRAFI plane}

The existence of a flat slice region is not immediately obvious but can be grasped as follow. Near the axis of the magnet coil, the lines of constant field are concave close to the magnet whereas, they are convex further away (Fig. 1B). By continuity, and because of the cylindrical symmetry of the field, an intermediate region must exist where the constant field lines are planar. This region can be precisely located by numerical simulations when the magnetic field is well known [21]. In what follows, a single loop of radius $a$ travelled by a current $I$ is first considered as a relevant example. Because of the cylindrical geometry, the magnetic induction can be decomposed in its axial and radial components of $H_{z}$ and $H_{\rho}$ :

$$
\begin{aligned}
& H_{z}(\rho, z)=\frac{I k}{4 \pi \sqrt{a \rho}}\left[l_{1}(k)+\frac{a^{2}-\rho^{2}-z^{2}}{(a-\rho)^{2}+z^{2}} l_{2}(k)\right] \\
& H_{p}(\rho, z)=\frac{I k z}{4 \pi \sqrt{a \rho}}\left[-l_{1}(k)+\frac{a^{2}+\rho^{2}+z^{2}}{(a-\rho)^{2}+z^{2}} l_{2}(k)\right]
\end{aligned}
$$

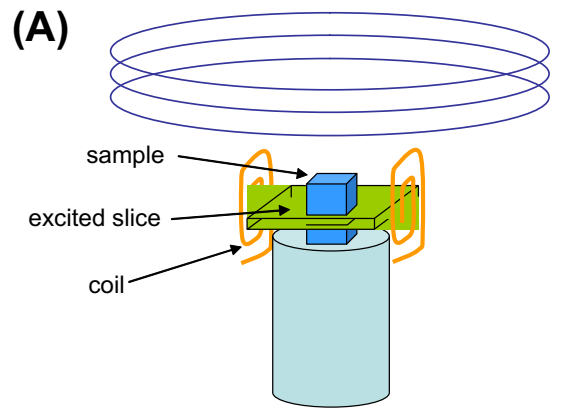

where $l_{1}$ and $l_{2}$ are Legendre elliptic integrals of the first and second kind of modulus $k$ :

$$
k=\sqrt{\frac{4 a \rho}{(a+\rho)^{2}+z^{2}}}
$$

Fig. 2 shows the numerical computation of the lines of constant field generated by a loop of radius $a$ perpendicular to the $z$ direction. In this figure, one can easily see that, at a distance about $z / a=0.6$ from the center of the coil, the curvature of the constant field lines change sign. This means that near this position, the lines of constant field are flat and perpendicular to the $z$ axis.

Beyond this simple example and in the general case, the position where this change of curvature takes place can be approached analytically through an expansion of the magnetic field around the $z$ axis. The magnetic induction obeys the following Maxwell equation:

$\operatorname{rot} \vec{H}=0$

Therefore, the magnetic induction can be derived from a scalar potential $V^{*}$. Close to the $z$ axis, this potential can be developed as a Taylor series near $\rho=0$. Considering the cylindrical geometry and Eq. (6), one can show that [21]:

$$
\begin{aligned}
V^{*}(\rho, z)= & V^{*}(0, z)-\frac{\rho^{2}}{4} \frac{d^{2} V^{*}(0, z)}{d z^{2}}+\frac{\rho^{4}}{64} \frac{d^{4} V^{*}(0, z)}{d z^{4}}+\ldots \\
& +\frac{(-1)^{n}}{(n !)^{2}}\left(\frac{\rho}{2}\right)^{2 n}\left(\frac{d}{d z}\right)^{2 n} V^{*}(0, z)+\ldots
\end{aligned}
$$

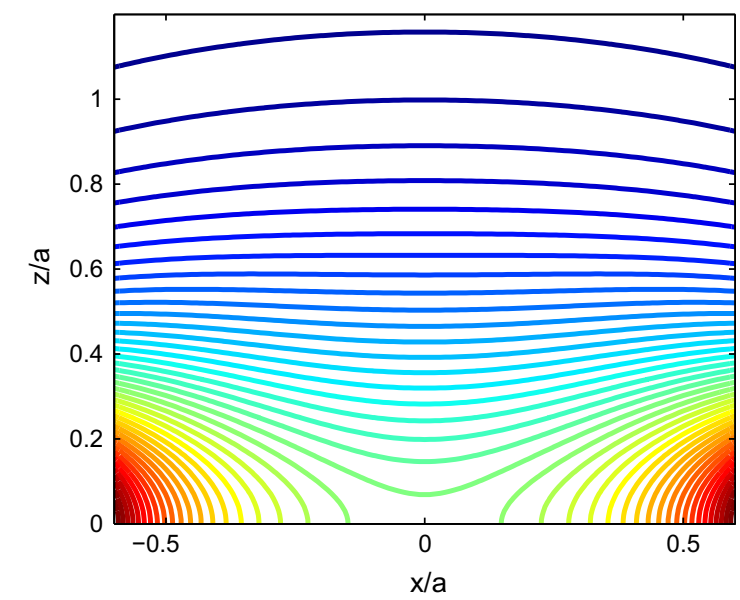

(B)

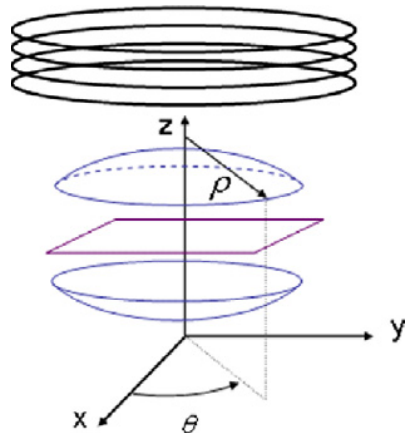

Fig. 1. (A) Sketch of the STRAFI experiment. (B) Sketch of the constant field lines at the onset of the magnet coil above and below the STRAFI plane. 
As $\vec{H}=-\operatorname{grad} V^{*}$, the axial and radial components $H_{z}$ and $H_{\rho}$ of the magnetic induction can be easily obtained:

$$
\begin{aligned}
H_{z}(\rho, z)= & \underline{H}(0, z)-\frac{\rho^{2}}{4} \frac{d^{2} \underline{H}(0, z)}{d z^{2}}+\frac{\rho^{4}}{64} \frac{d^{4} \underline{H}(0, z)}{d z^{4}}+\ldots \\
& +\frac{(-1)^{n}}{(n !)^{2}}\left(\frac{\rho}{2}\right)^{2 n}\left(\frac{d}{2}\right)^{2 n} \underline{H}(0, z)+\ldots \\
H_{\rho}(\rho, z)= & -\frac{\rho}{4} \frac{d \underline{H}(0, z)}{d z}+\frac{\rho^{3}}{16} \frac{d^{3} \underline{H}(0, z)}{d z^{3}}+\ldots \\
& +\frac{(-1)^{n}}{n !(n-1) !}\left(\frac{\rho}{2}\right)^{2 n-1}\left(\frac{d}{d z}\right)^{2 n-1} \underline{H}(0, z)+\ldots
\end{aligned}
$$

where $H(\rho, z)$ is the modulus of the field. Note that by symmetry, $H_{\rho}(0, z)=0$ and thus $H(0, z)=H_{z}(0, z)$.

At this point, one should keep in mind that in NMR, spins are sensitive to the modulus of the magnetic field which makes them rotate at the Larmor frequency. Thus, to get a planar selected slice, the radial variation of the modulus of the field must be zero. It is often claimed that the highest resolution is obtained at the inflection point of the modulus of the magnetic field, namely at the position $z_{\text {inflexion }}$ where

$$
\frac{d^{2} \underline{H}\left(0, z_{\text {inflexion }}\right)}{d z^{2}}=0
$$

Eqs. (8) and (9) show this is not strictly true. Certainly, at this point, the $z$ component of the field is radially constant up to fourth order. However, at the inflection point, the absolute value of the first derivative is not only non zero but even at its maximum. Thus, one must consider instead the development of the square modulus of the induction:

$$
\begin{aligned}
H_{\rho}^{2}+H_{z}^{2} & =\underline{H}^{2}(0, z) \\
& +\rho^{2}\left(-\frac{1}{2} \underline{H}(0, z) \frac{d^{2} \underline{H}(0, z)}{d z^{2}}+\frac{1}{4}\left(\frac{d \underline{H}(0, z)}{d z}\right)^{2}\right) \\
& \left.+\frac{\rho^{4}}{16}\left(\frac{d^{2} \underline{H}(0, z)}{d z^{2}}\right)^{2}+\frac{1}{2} \underline{H}(0, z) \frac{d^{4} \underline{H}(0, z)}{d z^{4}}-\frac{d \underline{H}(0, z)}{d z} \frac{d^{3} \underline{H}(0, z)}{d z^{3}}\right)+\cdots
\end{aligned}
$$

To obtain the highest possible resolution on a large sample ( $\rho$ as large as possible), the second order term has to be zero. So, rather than trying to fulfill (10), one should look instead for the position $z_{0}$, defined as the solution of the differential equation:

$$
-\frac{1}{2} \underline{H}\left(0, z_{0}\right) \frac{d^{2} \underline{H}\left(0, z_{0}\right)}{d z^{2}}+\frac{1}{4}\left(\frac{d \underline{H}\left(0, z_{0}\right)}{d z}\right)^{2}=0
$$

where $\underline{H}(0, z)$ is a function of the magnet geometry. This position can be easily identified for simple geometries where this function $H(0, z)$, that is the induction on the $z$ axis, is well known. In our illustrative case of the simple loop, the induction on the loop axis is:

$\underline{H}(0, z)=\frac{I a^{2}}{2\left(z^{2}+a^{2}\right)^{3 / 2}}$

In that case, the solution to Eq. (12) is

$$
z_{0}=\frac{\sqrt{10}}{5} a \sim 0.632 a
$$

which is significantly different from the location of the inflexion point:

$$
z_{\text {inflexion }}=\frac{1}{2} a=0.5 a \text {. }
$$

Yet, for a single loop, the gradient at $z_{0}$ is still $95 \%$ of its maximum value located at the inflexion point. This means that by positioning the sample at $z_{0}$ instead of at the inflexion point, it is still possible to benefit from a large gradient but with a much better resolution.

This last result was obtained analytically for the particular case of a single loop. However, to derive Eq. (12), no particular constraint was put on the geometry of the superconducting coil except the assumption of a cylindrical symmetry. Consequently, this equation stands even for more complex geometries like the ones of NMR or MRI superconducting magnets. It is thus concluded that the optimum position for a STRAFI experiment is not the inflexion point. Another important conclusion is that, to determine the position of the STRAFI plane, the sole requirement is the knowledge of the modulus of the magnetic field along the main axis of the magnet. This knowledge can be easily obtained experimentally by moving a sample along the $z$ axis and measuring its NMR Larmor frequency.

\section{Experimental}

In a STRAFI experiment, the coil has to fulfill several conditions to allow high resolution profiling of materials within reasonable times. First, it has to be located as close as possible to the excited slice which is often very thin. Second, it has to be compatible with the cylindrical geometry. Finally, it should also withstand the power associated with the use of very short pulse lengths. Keeping in mind these constraints, a custom-made double saddle coil was built based on the calculations of Javelot's thesis [22] and circuits designed by Mispelter [23]. This coil has a diameter of $25 \mathrm{~mm}$ and a height of $15 \mathrm{~mm}$.

Concerning the sample, it is placed at the top of a rod which transmits the motion from the stepper motor. In this particular design, the sample can be moved independently from the coil which is fixed at the STRAFI plane location. This fixed-coil design was chosen to insure that the sensitive layer is always excited in the same way. The custom-made probe of this study is depicted in Fig. 3.

A Carr Purcell Meiboom Gill (CPMG) sequence was used in all experiments because it can be used in a strong gradient [24-26]. Unless stated otherwise, the pulse length was $6 \mu$ s and, depending on the $T_{2}$, between ten and a few hundred echoes were acquired and summed. The time between the two first pulses was $20 \mu \mathrm{s}$. As a result, the echo time was $49 \mu \mathrm{s}$.

The superconducting magnet we used is the wide bore $7 \mathrm{~T}$ magnet of a Bruker Avance III spectrometer. A custom-written Labview $^{\circledR}$ program controlled the Bruker spectrometer to allow realtime processing of the data and to insure synchronization of the NMR acquisition with the motion.

\section{Results and discussion}

\subsection{Mapping of the axial component of the field}

The magnetic induction along the $z$ axis was mapped by moving a $2 \mathrm{~mm}^{3}$ piece of rubber along this axis and recording the resonance frequency of the rubber protons at various $z$ values. According to the conclusion of the theoretical section above, the differential Eq. (12) must be solved to determine where in the stray field the resolution is the highest. For this purpose, the experimental values of the dependence of the modulus of the magnetic induction with the axial position must be reduced to a functional form. The exact geometry of a given superconducting magnet is generally not known by its users. Still, it is reasonable to approximate its magnetic induction by the one delivered by a simple solenoid magnetic induction. Accordingly the magnetic induction can be modeled by the following form [21]: 


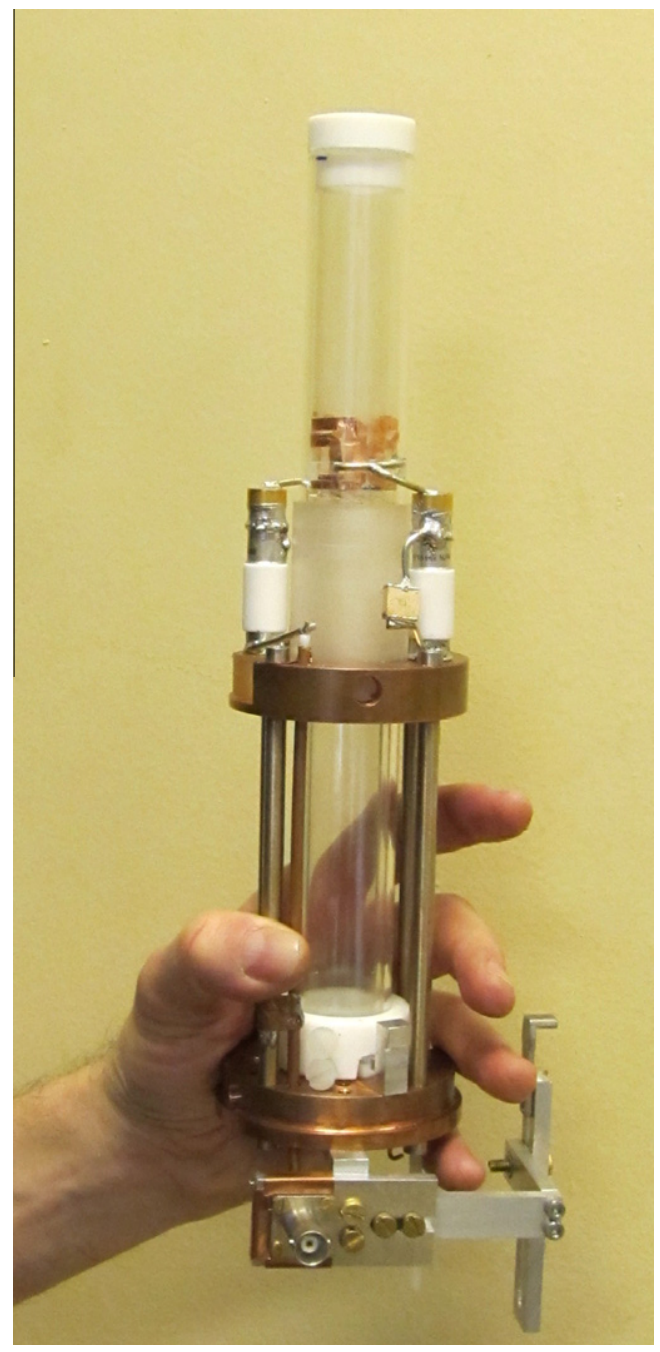

Fig. 3. Picture of our home-made STRAFI probe. The coil is made of copper strips sticked to a glass tube which crosses the whole probe. Thus, the sample positioned on top of a rod can move freely inside the glass tube whereas the probe is fixed against the superconducting magnet.

$\underline{H}(0, z)=\frac{I}{2 p}\left[\frac{N \cdot p / 2+z}{\sqrt{a^{2}+(N \cdot p / 2+z)^{2}}}+\frac{N \cdot p / 2-z}{\sqrt{a^{2}+(N \cdot p / 2-z)^{2}}}\right]$

Again, usually, the parameters I (the current), $p$ (the path of the helix defining the solenoid), $a$ (its radius) and $N$ (the number of loops) are not known. Furthermore, it must be understood that, the exact geometry of the superconducting magnet might not be a simple solenoid and that these parameters represent effective physical quantities. They have to be obtained by fitting this functional form to the experimental values. Then, the solution to Eq. (12) for this function effectively describing the induction was searched numerically. For the magnet of the present study, the STRAFI plane was found at $24.4 \mathrm{~cm}$ from the center of the magnet (see Fig. 4). The corresponding field strength and gradients at the STRAFI position were $2.97 \mathrm{~T}$ and $50.4 \mathrm{~T} / \mathrm{m}$ respectively (to be compared to $3.7 \mathrm{~T}$ and $53.6 \mathrm{~T} / \mathrm{m}$ at the inflexion point which was located at $23.0 \mathrm{~cm})$. Accordingly, the STRAFI plane had to be searched near $24 \mathrm{~cm}$ from the center of the magnet.

\subsection{Mapping of the radial component of the field}

Starting from the approximate position of the STRAFI plane obtained as explained above, the radial component of the magnetic

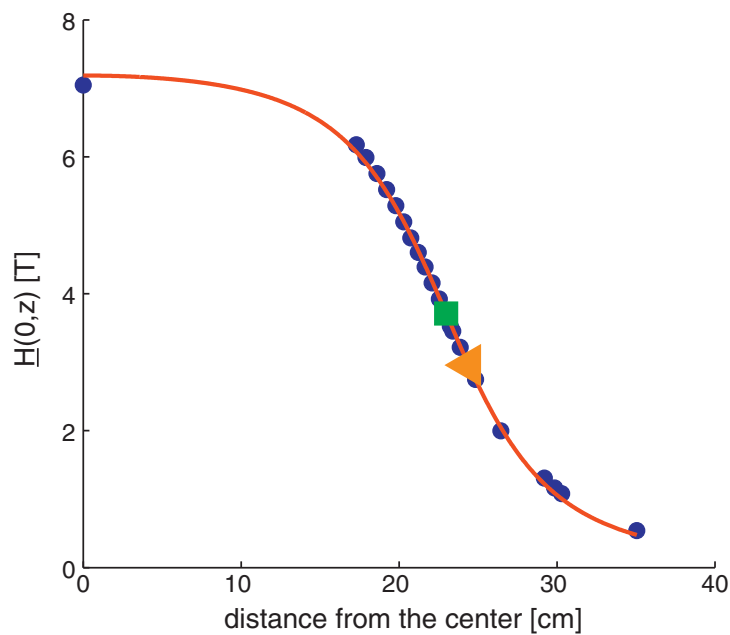

Fig. 4. Amplitude of the axial component of the magnetic induction of the superconducting magnet (points) used in this study and fit with a solenoid model (line) according to Eq. (16) (the fitting coefficients are $\mathrm{I} / 2 \mathrm{p}=3.7556 \mathrm{~A} / \mathrm{m}, \mathrm{a}=0.0698$ $\mathrm{m}$ et $\mathrm{N} . \mathrm{p} / 2=0.2298 \mathrm{~m}$ ). The square represents the inflexion point and the left pointing triangle the theoretical position of the STRAFI plane of the solenoid model.

field was mapped by moving a thin sample in the radial direction and recording its change in frequency. The sample used was a $2 \mathrm{~mm}$ wide spot of beauty cream between two glass slides. The interest of this beauty cream is that the wetting properties of the glass by this emulsion results in the spontaneous formation of a film with a definite thickness of $10 \mu \mathrm{m}$ over an extension of a few millimeters. The probe custom-made for the present study allowed the excitation of slices as thin as $100 \mu \mathrm{m}$ when necessary (pulse length of $5 \mu$ s in a gradient of about $50 \mathrm{~T} / \mathrm{m}$ ).

Fig. 5 shows the result of the radial mapping of the field at various $z$ positions around $24 \mathrm{~cm}$ from the center in the superconducting magnet. For each frequency, the thin sample was first centered within the magnet. Then, it was radially moved and its frequency change recorded. Note that with this method, the curvature is inverted against the sketch of Fig. $1 \mathrm{~B}$ where the lines of constant field are represented. The position where the curvature is zero was located at $137 \mathrm{MHz}$ which corresponds to a magnetic field of

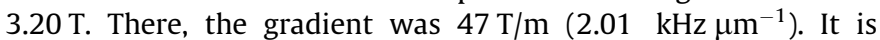
remarkable that this position was only $5 \mathrm{~mm}$ higher than the approximate position predicted by simply measuring the magnetic field along the $z$ axis as exposed in the previous section.

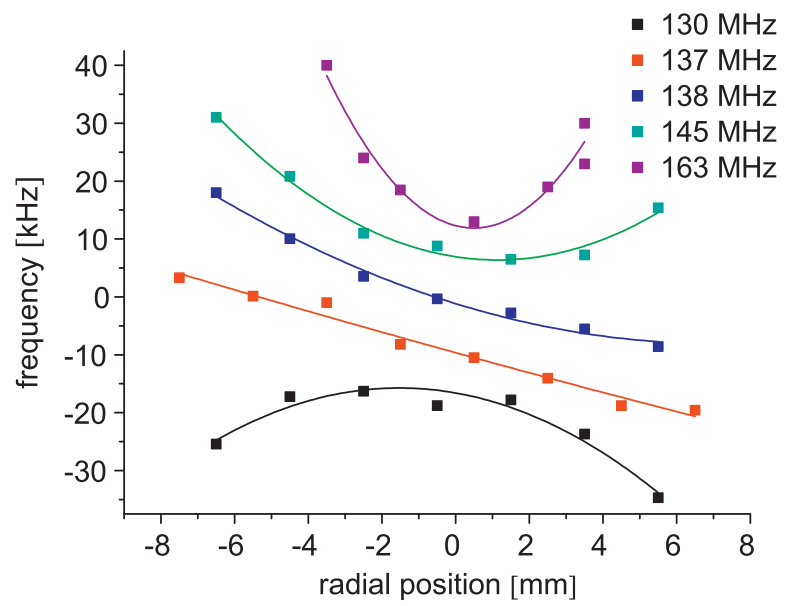

Fig. 5. Radial mapping of the magnetic field at various heights in the magnet. The figure is the plot of the frequency shift from a given base frequency (axial position) resulting from an off centered lateral motion. The acquisition protocol is described in Section 4.1 . 
Even if the frequency where the isofields are planar is now determined, the curves were still tilted due to a misalignment of the STRAFI plane with respect to the one within which the sample was moved.

\subsection{Alignment of the sample}

One of the key points of the STRAFI experiment concerns the resolution that can be obtained on a wide sample. Consequently, the next step to reach the ideal configuration depicted in Fig. 1 is to correct the tilt between the sample holder and the STRAFI plane. The working frequency is the one determined in the above Section 4.2 to minimize the curvature of the field, namely $137 \mathrm{MHz}$. The method used to adjust the sample holder tilt is the following. A "spot" of cream ( $2 \mathrm{~mm}$ diameter and $10 \mu \mathrm{m}$ of thickness) is placed off-center on a rotational stage with an axis roughly perpendicular to the STRAFI plane. The spot is then brought within the STRAFI plane using a vertical stage. The signal of the spot is obtained with an $8 \mu$ s pulse duration which corresponds to an excited slice of $60 \mu \mathrm{m}$. It has been seen previously that changing the $z$ position of the spot inside the STRAFI plane induces a change of its resonance frequency. The frequency shift (in the kiloHertz range) as a function of the angle described by the rotational stage is recorded (Fig. 6). If there were no tilt between the plane of rotation of the sample and the STRAFI plane, the rotation would induce no $z$ position variation of the spot and thus no frequency shift. The vertical stage axis was thus tilted to minimize the frequency dependence upon rotation of the sample. Proceeding iteratively, one should ideally reach a situation where the STRAFI plane fixed by the position of the superconducting magnet is aligned with the sample plane.

On Fig. 6, the frequency dependence of the spot moving on a circle of $2 \mathrm{~cm}$ is $15 \mathrm{kHz}$. The value of the gradient whose magnitude is $2.01 \mathrm{kHz} . \mu \mathrm{m}^{-1}$ yields a resolution of $4.25 \mu \mathrm{m}$ on a $1 \mathrm{~cm}$ wide sample. This resolution constitutes probably a limit beyond which other experimental complications arise, such as the thermal expansion of the sample holder.

\subsection{Resolution test}

The phantoms used to test the resolution on actual samples were made of cream films pressed between glass plates. The interesting feature of these test samples is that the planarity of the glass plates is well below micrometric specifications. Furthermore, the thickness of the cream films is remarkably constant and reproducible as it is fully determined by its glass wetting properties, regard-

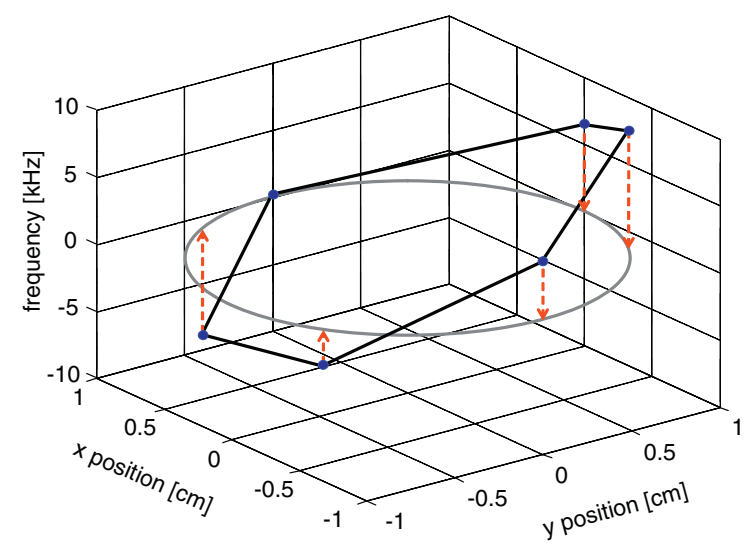

Fig. 6. Frequency shift $(\mathrm{kHz})$ of an off-centered cream "spot" (1 mm diameter, $10 \mu \mathrm{m}$ thickness) versus the position of the sample holder (see Section 4.3 ). The position of the spot is $1 \mathrm{~cm}$ from the center. The tilt angle between the STRAFI plane and the sample plane is $8 \times 10^{-4} \mathrm{rad}$. less of variations in the preparation procedure. The lateral extension of the films can be varied at will by controlling the amount of cream deposited between the glass plates. With this configuration, it is possible to check the resolution on a centimeter-wide sample. Fig. 7A displays the profile of a multilayer sample made with three $1 \mathrm{~cm}$ diameter glass plates. A first off-centered cream "spot" layer ( $3 \mathrm{~mm}$ diameter) was pressed between the two first plates and a second $1 \mathrm{~cm}$ wide cream layer was pressed between the second and the third plate (Fig. 7B). The first peak corresponded to the wide layer. The intensities were not equal as they reflect the different volumes of the intersection of the STRAFI plane with the successive layers of the sample, and thus depended on the diameter of the two layers ( $1 \mathrm{~cm}$ versus $3 \mathrm{~mm}$ ). To acquire the profile, the $z$-axis motor was moved step by step in increments of $5 \mu \mathrm{m}$. Taking into account the magnitude of the gradient $(50.4 \mathrm{~T} /$ $\mathrm{m})$, the square pulse of duration $100 \mu$ s excited a $5 \mu \mathrm{m}$ thick slice (Eq. (2)). The two layers are separated by a distance of $150 \mu \mathrm{m}$ corresponding to the thickness of the intermediate glass plate. The thickness of each cream layer is $10 \mu \mathrm{m}$.

It must be recognized that profiling with a step-by-step motion has the definite advantage of allowing to profile a sample not only with very small steps but also over a large course (a few $\mathrm{cm}$ ). As an illustration, the profile of a sample made of as many as seven layers of cream films separated by $150 \mu \mathrm{m}$ thick glass slides was recorded (Fig. 8A). It was done step by step with steps size of $10 \mu \mathrm{m}$ and over a range of $3 \mathrm{~mm}$. Fig. $8 \mathrm{~B}$ displays a more precise profile of part of the multilayer sandwich at the position $21.18 \mathrm{~mm}$, which was obtained with a finer step size of $5 \mu \mathrm{m}$ and a pulse duration of $100 \mu \mathrm{s}$. It is thus shown that it is indeed possible to obtain a complete profile on a large scale while maintaining, when needed, a

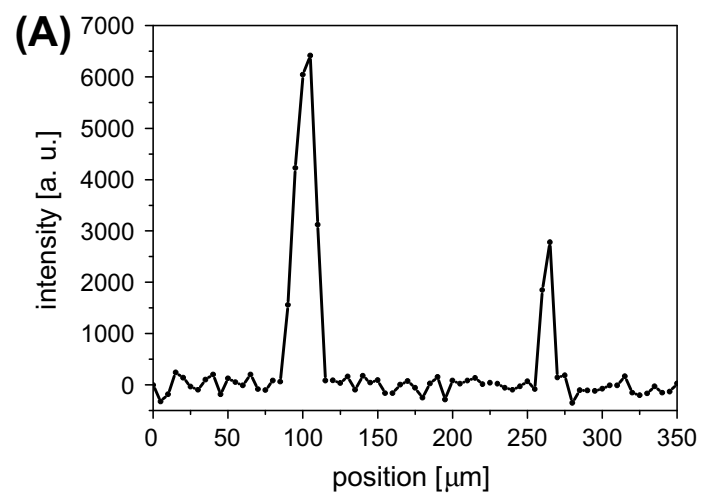

(B)

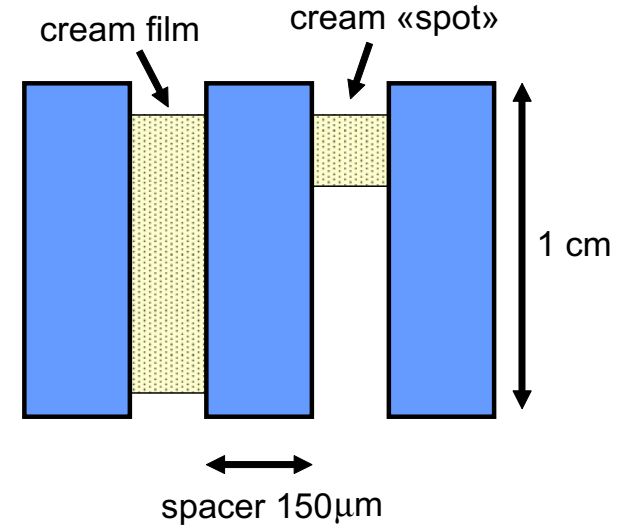

Fig. 7. (A) Step-by-step ( $5 \mu \mathrm{m}$ step) profile of a bilayer phantom two cream films separated by a $150 \mu \mathrm{m}$ glass slide. (B) Sketch not to scale of the bilayer phantom profiled in (A). The first film is $1 \mathrm{~cm}$ wide. Experimental details are given in Section 4.4. The total acquisition time is 45 minutes. 


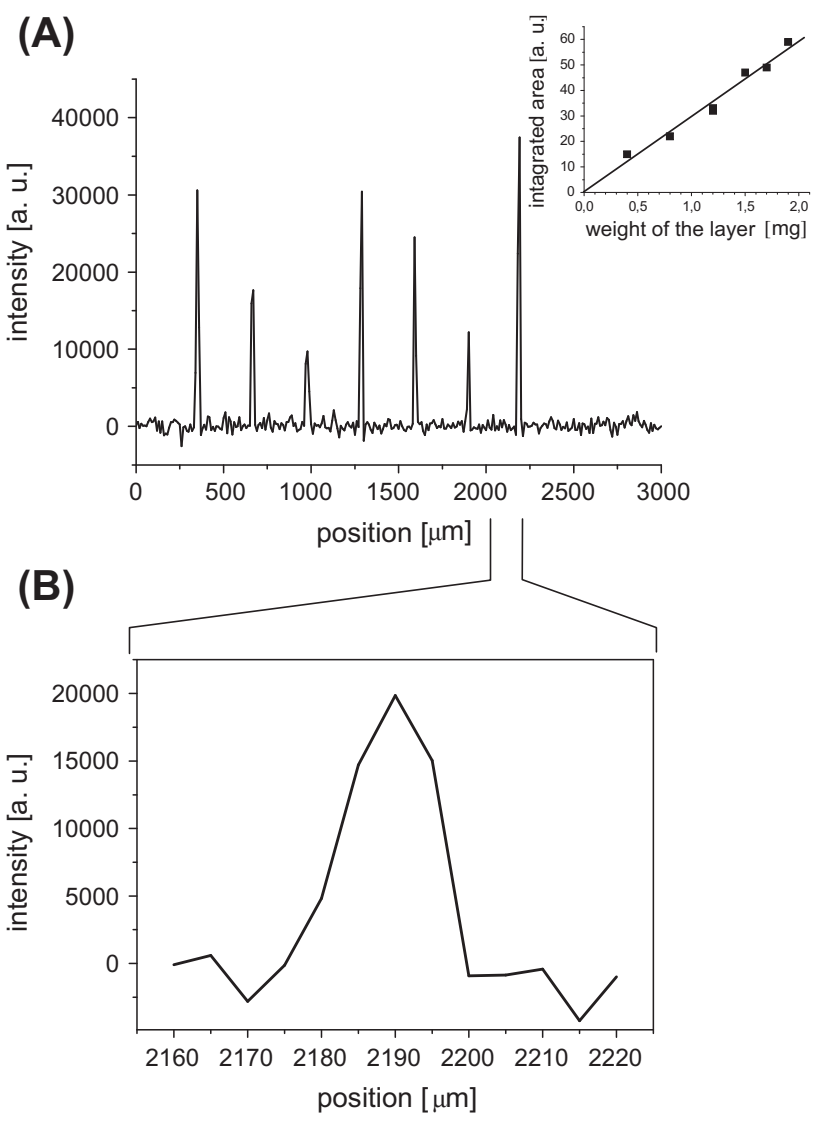

Fig. 8. (A) Step-by-step profile of a thin film cream multilayer. Each $1 \mathrm{~cm}$ wide film is separated by a glass slide of $150 \mu \mathrm{m}$ thickness. The step is $10 \mu \mathrm{m}$ (B) Step-by-step profile of the film of the 7 th peak of the (A) multilayer. The step size is $5 \mu \mathrm{m}$. Experimental details are given in Section 4.4.

resolution of $5 \mu \mathrm{m}$. Let us emphasize that the amplitude variations were not artifacts but reflected the actual lateral extensions of the films. The amount of cream in each layer can be appreciated by measuring their weights. As it turned out, for each peak, the integrated intensities correlated well with the weight of the corresponding layers.

In the step-by-step profiling experiments, the best resolution was obtained by excitation of a $5 \mu \mathrm{m}$ thick slice with pulses of duration $100 \mu \mathrm{s}$. Taking into account the inter-pulse delay of $100 \mu \mathrm{s}$, the echo time was thus $350 \mu$ s. Such long pulses and echo time are clearly not compatible with the measurement of fast relaxing materials with $T_{2}$ 's typically below $100 \mu \mathrm{s}$. In that case, to achieve the same $5 \mu \mathrm{m}$ resolution, STRAFI profiling must be combined with Fourier Imaging [27]. Then, the fast relaxing species are excited using short pulses duration and short inter-pulse delays ( 4 and $10 \mu \mathrm{s}$, respectively, in the present case). The echo time is thus reduced to less than $30 \mu \mathrm{s}$. Subsequently, the spatial decoding is obtained, not by moving the sample as previously, but by Fourier transformation of the echo. As a consequence, the spatial resolution is now proportional to the spectral resolution. Here, with a gradient of $2.01 \mathrm{kHz} / \mu \mathrm{m}$, the $5 \mu \mathrm{m}$ resolution corresponded to a separation of $10 \mathrm{kHz}$, a value which can be resolved with $T_{2}$ 's as low as $70 \mu \mathrm{s}$. Consequently, in principle, combining Fourier imaging to STRAFI is potentially useful for materials profiling as it allows maintaining the same resolution on samples while significantly shortening the observable $T_{2}$ 's.

To check that the resolution obtained by Fourier Imaging is indeed similar to the one obtained by step-by-step motion, the corresponding experiment was performed on the same type of bilayer

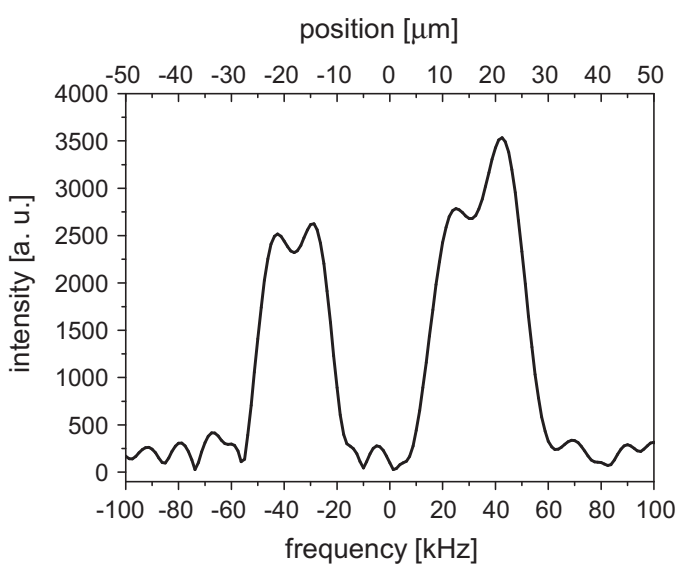

Fig. 9. Fourier transform of the echo obtained on a bilayer made of two thin films separated by a thin spacer of $20 \mu \mathrm{m}$. The pulse length was of $4 \mu$ s (see Section 4.4 for experimental details).

cream sample. Again, in this experiment, the sample was kept at a fixed position in the STRAFI plane, in contrast to all previous results obtained by moving the object with a stepper motor. The limitation of the observation window of the Fourier transform profile arises from the intensity of the gradient. With a gradient magnitude of $2.01 \mathrm{kHz} . \mu \mathrm{m}^{-1}$, a hard pulse of $4 \mu$ s excites a slice of $125 \mu \mathrm{m}$. This allows the observation of a bilayer made of two thin films separated by a thin spacer of $20 \mu \mathrm{m}$. A Zero-filling to 1024 points followed by a Fourier transformation provided the profile of Fig. 9. Compared to the other plots, the profile appears smoother, which is an effect of the zero-filling. Then, it is easier to deduce the resolution from the derivative of the curve. A width of $10 \mathrm{kHz}$ was obtained in the derivative of the profile, corresponding to a resolution of $5 \mu \mathrm{m}$. It is thus verified that the same resolution can be obtained, either with soft pulses and step-by step sample motion, or by hard pulses and Fourier Imaging.

\section{Conclusion}

Up to now, STRAFI experiments have succeeded in profiling samples but without achieving the micrometer resolution expected of a gradient in the range of $50 \mathrm{~T} / \mathrm{m}$, probably because of ill-positioning and misalignment of the sample in the fringe field. In this study, the STRAFI plane was precisely located relatively to the inflexion point of the field and it was established that both do not coincide. A major criterion for a useful profiling tool is to be able to obtain a good axial resolution on samples with a large lateral extension. In this regard, by accurately mapping the field magnitude around the magnet axis, we have shown that it was possible to profile a sample with a typical size of $1 \mathrm{~cm}$ (width and height) with a resolution as good as $5 \mu \mathrm{m}$. This geometry appears particularly adapted to the study of multilayered materials or for the study of transport phenomena at liquid/solid of liquid/ gas interfaces. Furthermore, in principle, when combined with Fourier Imaging, the STRAFI method can achieve the same micrometer-scale resolution on fast relaxing solid materials. This last point will be the object of a forthcoming communication [28].

\section{Acknowledgments}

This work is supported by Saint-Gobain Recherche. We would like to gratefully thank J. Mispelter (INSERM, Institut Curie, France) and D. Petit (CNRS, Ecole Polytechnique, France) for their help in designing our probe. We also thank J. Perlo and F. Casanova (RWTH Aachen, Germany) for their advice. 


\section{References}

[1] P. Lauterbur, Image formation by induced local interactions - examples employing nuclear magnetic-resonance, Nature 242 (1973) 190-191.

[2] P. Mansfield, P.K. Grannell, Diffraction and microscopy in solids and liquids by NMR, Phys. Rev. B 12 (1975) 3618-3634.

[3] J.F.P. Ullmann, G. Cowin, S.P. Collin, Magnetic resonance microscopy of the barramundi (Lates calcarifer) brain, J. Morphol. 271 (2010) 1446-1456.

[4] S.C. Lee, K. Kim, J. Kim, S. Lee, J.H. Yi, S.W. Kim, K.S. Ha, C. Cheong, One micrometer resolution NMR microscopy, J. Magn. Reson. 150 (2001) 207-213.

[5] A.A. Samoilenko, D.Y. Artemov, L.A. Sibeldina, Formation of sensitive layer in experiments on NMR subsurface imaging of solids, JETP Lett. 47 (1988) 417419.

[6] S.G. Pereira, R. Fulgencio, T.G. Nunes, M. Toledano, R. Osorio, R.M. Carvalho, Effect of curing protocol on the polymerization of dual-cured resin cements, Dent. Mater. 26 (2010) 710-718.

[7] H. Stork, A. Gadke, N. Nestle, F. Fujara, Flat RF coils in static field gradient nuclear magnetic resonance, J. Magn. Reson. 200 (2009) 321-327.

[8] T.G. Nunes, E.W. Randall, G. Guillot, The first proton NMR imaging of ice: strayfield imaging and relaxation studies, Solid State Nucl. Magn. Reson. 32 (2007) 59-65.

[9] S.G. Pereira, N. Reis, T.G. Nunes, Spatially resolved studies on the photopolymerization of dimethacrylate monomers, Polymer 46 (2005) 80348044.

[10] J. Boguszynska, M.C.A. Brown, P.J. McDonald, J. Mitchell, M. Mulheron, J. TrittGoc, D.A. Verganelakis, Magnetic resonance studies of cement based materials in inhomogeneous magnetic fields, Cem. Concr. Res. 35 (2005) 2033-2040.

[11] A.J. Bohris, U. Goerke, P.J. McDonald, M. Mulheron, B. Newling, B. Le Page, A broad line NMR and MRI study of water and water transport in Portland cement pastes, Magn. Reson. Imaging 16 (1998) 455-461.

[12] B. Newling, D.R. Ward, V. Vijayakrishnan, Stray field magnetic resonance imaging (STRAFI) of water transport in ceramic substrates, J. Porous Mater. 8 (2001) 193-199.

[13] K.J. Carlton, M.R. Halse, J.H. Strange, Diffusion-weighted imaging of bacteria colonies in the STRAFI plane, J. Magn. Reson. 143 (2000) 24-29.
[14] I. Hopkinson, R.A.L. Jones, S. Black, D.M. Lane, P.J. McDonald, Fickian and case II diffusion of water into amylose: a stray field NMR study, Carbohydr. Polym. 34 (1997) 39-47.

[15] A.A. Samoilenko, D.Y. Artemov, L.A. Sibeldina, Application of the NMRintrascopy in the study of an interior structure of solids, Zh. Fiz. Khim. 61 (1987) 3082-3085.

[16] J.H. Iwamiya, S.W. Sinton, Stray-field magnetic resonance imaging of solid materials, Solid State Nucl. Magn. Reson. 6 (1996) 333-345.

[17] K. Zick, STRAFI Solids Imaging, Technical Report NMR/B353/393, Bruker Anlytische Messtechnik GmbH Silberstreifen, D-7512 Rheinstetten 4/ Karlsruhe, 1993.

[18] P.J. McDonald, Stray field magnetic resonance imaging, Prog. Nucl. Magn. Reson. Spectrosc. 30 (1997) 69-99.

[19] P.J. McDonald, B. Newling, Stray field magnetic resonance imaging, Rep. Prog. Phys. 61 (1998) 1441-1493.

[20] J. Mitchell, P. Blumler, P.J. McDonald, Spatially resolved nuclear magnetic resonance studies of planar samples, Prog. Nucl. Magn. Reson. Spectrosc. 48 (2006) 161-181.

[21] E. Durand, Electrostatique et Magnétostatique, Masson \& Cie, Paris VI, 1953.

[22] S. Javelot, Réalisation d'un dispositif utilisant la RMN pour caractériser les écoulements multiphasiques, Université Pierre et Marie Curie, Paris VI, 1994.

[23] J. Mispelter, M. Lupu, A. Briguet, NMR Probeheads for Biophysical and Biomedical Experiments, Imperial College Press, London, 2006.

[24] M.D. Hürlimann, D.D. Griffin, Spin Dynamics of Carr-Purcell-Meiboom-Gilllike Sequences in Grossly Inhomogeneous B0 and B1 Fields and Application to NMR Well Logging, J. Magn. Reson. 143 (1) (2000) 120-135.

[25] Y.-Q. Song, Categories of Coherence Pathways for the CPMG Sequence, J. Magn. Reson. 157 (2002) 82-91.

[26] G. Goelman, M.G. Prammer, The CPMG pulse sequence in strong magneticfield gradients with applications to oil-well logging, J. Magn. Reson. Ser. A 113 (1995) 11-18.

[27] P.M. Glover, P.J. McDonald, B. Newling, Stray-field imaging of planar films using a novel surface coil, J. Magn. Reson. 126 (1997) 207-212.

[28] M. Van Landeghem, B. Bresson, J.-B. d'Espinose de Lacaillerie, Cem. Concr. Res, in preparation. 